\title{
DISONANCIAS ENTRE EL ENTORNO LOCAL DE LOS NEGOCIOS Y LA CULTURA CORPORATIVA EN UN PAÍS LATINOAMERICANO*
}

\author{
Margalit Berlin \\ Profesora de la Escuela de Estudios Sociales \\ de la Universidad Simón Bolívar, Venezuela
}

E ste ensayo estudia la relación entre la cuitura corporativa de una compañía multinacional con sede en Estados Unidos y el entorno y las práeticas de los negocios en Venezuela. La corporación estudiada se dedica a los servicios financieros y goza de mucho prestigio a nivel mundial, al que otorga gran importancia. Para que éste sc mantenga, la corporación cumple estrictamente las órdenes que provienen de su sede -en Nueva York-, impo ne una supervisión estrecha y rígida y proporciona una capacitación especializada y continua a su personal, tanto en Venezuela -por expertos internacionales-como en su sede u otras filiales importantes. La cultura que prevalece en la corporación es la norteamericana, y sus gerentes de mayor categoría provienen de Estados Unidos.

Por su parte, el contexto empresarial venezolano se caracteriza por la importan-

\footnotetext{
" Traducido del inglés por Federico Ruádez.
}

cia capital dada a los lazos familiares, lo que dificulta el que los gerentes locales de la corporación multinacional se adapten totalmente a la cultura de la organización. Al ir madurando y cambiando sus circunstancias personales, los gerentes localcs ya no se avienen a las metas de la corporación. pues ésta no puede ofrecerles la posibilidad de satisfacer sus necesidades y objetivos profesionales. Es en este momento cuando el entorno local de los negocios se les presenta como la alternativa y comienzan a pensar en dejar la corporación.

\section{El método}

La empresa objeto de este estudio se escogí́ tomando en cuenta los objetivos de la investigación: analizar la interacción entre el entorno local de los negocios y la cultura corporativa (criterios similares a los utilizados por Elsbach y Sutton, 1992, y Dutton y Dukerich, 1992), asícomo la accesihilidad para la investigadora (quicn csto escribe). 
Por casualidad, la corporación necesitaba una secretaria ejecutiva, cargo que esta investigadora podíadesempeñary que pudo conseguir con la recomendación de un viejo amigo, un gerente de alto nivel de esta misma corporación.

\section{Fuentes de datos}

La principal herramienta utilizada fue la observación participante (durante cuatro meses). Como secretaria ejecutiva, la investigadora debía trabajar con dos gerentes internacionales que venían periódicamente a Venezuela para realizar auditorías a dos departamentos (ambos fueron parte de la muestra de seis informantes). Su presencia diaria en la corporación y la naturaleza de sus funciones le permitió observar a los sujetos de la organización. analizar su comportamiento y forma de relacionarse entre ellos $-\mathrm{y}$ hasta el espacio físico donde esto se producía-. También observar los sistemas de comunicación, supervisión y entrenamiento, e identificar las tradiciones y los sutiles códigos de conducta y valores que tienen vigencia en la corporación.

\section{Observación de hechos especiales}

Como ya se mencionó, la investigadora tuvola oportunidad de observar la auditoría realizada a dos departamentos, trabajo que anualmente llevan a cabo gerentes internacionales que vienen periódicamente con ese propósito. La conducta de los gerentes y el personal en esa ocasión reveló algunos de los valores más importantes compartidos por la empresa, tales como el valor que se le otorga al trabajo en equipo.

Los departamentos auditados y su ge- rente se evalúan por su capacidad para trabajar en equipo y según como aplican un sistema rígido de supervisión diseñado en la sede para decidir las oportunidades de negocios. A fin de proteger el código de ética de la empresa, los gerentes deben regirse por ciertos criterios ya establecidos para seleccionar a los clientes, cn lugar de utilizar sus propios criterios y, eventualmente, favorecer sus intereses personales.

\section{Entrevistas semiestructuradas}

Se aplicó entrevistas estructuradas a seis gerentes para obtener información más organizada en relación con temas especifi$\cos$. La investigadora indagó acerca de los valores de la corporación, es decir, las características de conducta más valoradas o condenadas por la empresa en cuanto a su orientación hacia los clientes, estilo de comunicación, supervisión, desarrollo de carreras, asícomo el grado de compromiso con la empresa.

Los datos obtenidos mediante las entrevistas complementaron los datos recogidos a través de la observación y permitieron formarse una idea de las perspectivas de los informantes. Estas respuestas fueron luegoclasificadas según edad, sexo, estado civil, responsabilidades familiarcs, y sirvieron para elaborar un perfil personal de cada uno de los gerentes informantes.

Los gerentes seleccionados para esta mucstra fueron los más accesibles; la investigadora trabajaba directamente con algunos de ellos. La relación más rápida se estableció con el gerente que la recomendó para el puesto, quien dirigía uno de los departamentos cuya auditoría observó. Se conocían desde niños, habían estudiado en la misma escuela primaria y en el 
bachillerato y tenían amigos comunes.

El vínculo con otras dos informantes fue facilitado porque la investigadora realizaba ejercicios aeróbicos junto con ellas durante el mediodía en el propio local de la empresa. Una de ellas era abogada de la firma; $y$ la otra, gerente de nivel medio de uno de los departamentos auditados.

Con otro gerente de nivel medio el víneulo se logro fácilmente porque esa persona estaba realizando estudios de posgrado en la universidad donde la investigadora trabajaba.

Las relaciones entabladas con los gerentes internacionales fueron más distantes, pues eran los jefes de la investigadora. Éstos portaban mucho de la cultura corporativa, ya que viajaban frecuentemente a Venezuela para asegurarse de que las órdenes provenientes de la sede fucran observadas estrictamente.

\section{La empresa y su cultura corporativa}

La empresa vende sus servicios en todo el mundo. Las relaciones de trabajo entre los empleados tienden a ser fluidas, con poca o ninguna formalidad. No es necesario concertar una cita para hablar con los ejecutivos medios y de alto nivel, solamente con el presidente. Los empleados están conectados por el correo electrónico; cada mañana el personal gerencial de la empresa se comunica a través de la computadora con Nueva York, Hong Kong o España.

Los nuevos empleados son entrenados permanentemente en el exterior para que aprendan y estén al tanto de los progresos tecnológicos. Es un proceso continuo que no termina después de los primeros meses.
Este tipo de entrenamiento internacional constituye una de las ventajas importantes que ofrece la empresa para promover un alto grado de compromiso organizacional.

Saber inglés es un requisito. Es común escuchar a los gerentes hablar en ese idioma en los pasillos. Se ofrecen cursos de inglés durante la hora del almuerzo para aquellos empleados que necesitan aprenderlo.

La supervisión se logra a través de una estructura burocrática rígida: las decisiones importantes se toman en la sede, en los Estados Unidos. Las reuniones de trabajo se realizan básicamente para discutir y distribuir las tareas entre los gerentes locales, no para discutir decisiones ya tomadas en la sede. Para cerrar cualquier trato de negocios -por lo menos se requieren dos firmas-. los gerentes dehen respetar el codigo de ética de la empresa.

La evaluación del personal la realizan supervisores extranjeros. Se basa en la evaluación del desemper̃o de los equipos đe trabajo y también tiene en cuenta la contribución individual; de esta manera estimula la identificación de la persona con su equipo de trabajo y con la empresa como un todo, Una evaluación positiva influye en los aumentos de sueldo y, eventualmente, en los ascensos de los miembros de los equipos. La supervisión internacional cumple otra función importante: ayuda al cumplimiento del código de ética de la corporación.

Los aspectos más valorados por la compañía son su naturaleza transnacional, la capacitación internacional que reciben los empleados, la supervisión y el rígido control burocrático que provienen de la sede, así como un código de ética que ha ayuda- 
do a la empresa a mantener una excelente reputación.

La conversación diaria entre los empleados gira en torno de asuntos comunes, como la inflación, el alto costo de la vida. las películas en cartelera; pero, sobre todo. trata acerea de la situación global de la compañía en el mercado.

Las noticias sobre las expansiones de la firma se transmiten diariamente a través del correo electrónico, contribuyendo a reforzar el sentido de pertenencia a la empresa. Mientras la investigadora estuvo aIlí escuchaba con frecuencia comentarios sobre los éxitos o fracasos recientes de la empresa. Por ejemplo, en una ocasión los gerentes expresaron su preocupación por el hecho de que un par de directivos valiosos para la firma aceptaron of crtas de trabajo en la competencia.

Esos dos discursos opuestos acerca de los éxitos de la firma y los terrenos que va ganando la competencia, accesibles a los empleados dentro de una organización, son lo que Tony Watson describe como un discurso sobre "facultar, destreza y crecimiento" y otro sobre "control, trabajos y costos": si bien el trabajador valora el hecho que el puesto desempeñado enriquece su carrera, también es consciente de que en otra empresa podría obtener una remuneración mayor en menos tiempoque el largo periodo que debe esperar en la corporación.

Cuando se va a contratar a un nuevo empleado, los funcionarios del departamento de personal señalan claramente que no debe existir conflicto alguno de intereses entre las metas de la corporación y las del candidato. Se espera que los empleados no favorezcan a clientes con quienes ten- gan lazos de amistad si el provecho de éstos puede no ser beneficioso para la compañía. Los gerentes demuestran que son de confianza al probar a la organización que ésta les importa más que cualquier otra cosa. Esto hace pensar en el estudio clásico de Kanter (1977: 53) sobre el personal de las corporaciones, quien explica que los gerentes que aparentan tener lealtades compartidas son considerados algo sospechosos. En ese estudio, una mala seña constituía, por ejemplo, que alguien del departamento de ventas se convirtiera en un "customer man" (hombre de los clientes), es decir, que complaciera a los clientes y se interesara por sus intereses antes que por los de la firma, aun cuando esto dabacomo resultado un incrementode las ventas.

En cste aspecto, la corporación estudiada es algo intlexible, aunque esto signifique ser poco práctica. Se arriesga a perder ejecutivos de alto nivel frustrados porque no pueden cerrar los tratos que han propuesto a la empresa.

El código de ética de la corporación es tan estricto que ha alejado a algunos de sus clientes en lugar de atraerlos. La firma cuenta con una especie de sistema de inteligencia que usa para revisar cuidadosamente las credenciales de los clientes, y se basa en éstc para cxcluir a algunos de ellos, aquienes son sospechosos de haber obtenido ganancias por medios ilícitos. Para algunos de los gerentes locales, la "lista negra" es elaborada con procedimientos arbitrarios, excluyendo a empresas o individuos que según su entender tienen derecho a ser clientes de esa prestigiosa empresa multinacional. Por esta razón, los gerentes que recomiendan a clientes posteriormente vetados se sienten frustrados, ya que esto no les permite obtener ganancias para 
la firma ni para ellos mismos (bonos). Los gerentes que sistemáticamente cierran tratos sin seguir el proceso de aprobación son sancionados indirectamente, pues no ascienden rápidamente.

Su código de ética le sirve a la firma para mantener su prestigio internacional, aun cuando opera en un entorno de negocios en el cual la corrupción es generalizada y forma parte de la vida cotidiana (Pérez-Perdomo, 1991). En Venezuela, una gran porción de la actívidad económica depende del gobierno, porque éste recibe la mayor parte del ingreso que entra al país a través de la venta del petróleo y, a su vez, lo redistribuye. Las empresas y personas que contratan con el gobierno generalmente cobran precios y honorarios más altosque los normales, ya sea por la venta de maquinaria o materia prima. o por el desarrollo de grandes proyectos de infraestructura. Este proceder, según la firma, es un medio ilegítimo de ganar dinero y por ello rechaza a determinados clientes, aunque a corto plazo obtenga menos ganancias.

Las entrevistas informales y conversaciones sostenidas durante el estudio mostraron que la adaptación a la cultura corporativa depende del perfil personal, los antecedentes educacionales, la edad, el estado civil y las cargas familiares.

Así, los gerentes venezolanos de origen extranjero con estudios de posgrado y numerosos contactos personales y familiares permanecían en la firma durante muchos años hasta alcanzar altos niveles dentro de la organización. Cuando ya no podían avanzar se retiraban para emprender sus propios negocios. Se adaptaban a la cultura organizacional porque desde una edad temprana aprendieron a admirar la cultura internacional.
Los gerentes que no tenían un nivel tan alto de educación o tantos contactos sociales se adaptaban a la firma debido al hecho de que valoraban trabajar para una firma internacional prestigiosa. En la época en que se realizó este estudio tenf́an unos 35 años de edad y sus ambiciones estaban dirigidas hacia el nivel gerencial más alto. No tenían intenciones de abandonar la firma, ya que, primero, no tenfan tantos contactos sociales $\mathrm{y}$, segundo, uno de ellos era una mujer con grandes responsabilidades familiares, lo que restringía su capacidad para asumir riesgos $y$, por lo tanto, sus oportunidades de emprender un negocio propio. El otro, un gerente venezolano que se graduó en la mejor universidad del pais, además de contar con una maestría en administración en la más renombrada escucla de posgrado en administración y tener contactos personales y familiares, no se adapt 6 a la supervisión rigida practicada por la firma. Se retiró después de seis meses (lapso que duró el entrenamiento que todos los gerentes deben recibir cuando ingresan a la firma) y empezó a trabajar en otra empresa multinacional, donde gozaba de inayor autonomía y más tiempo para cuitivar aquellos vínculos que tanto apreciaba y que son tan útiles en Venezuela.

Otro gerente venezolano con una educación promedio que se đesempeñaba muy bien renunció cuando encontró una posición en otra firma local que le ofrecía la oportunidad de desarrollar contactos personales y un mayor número de negocios. No estaba de acuerdo con el proceso de aprobación al que se sometía a todo cliente potencial. que contradecía su propia visión ética del país, basada en un conocimiento más profundo del entorno latinoamericano de los negocios que el que tiene una casa matriz ubicada en los Estados Unidos. 


\section{El entorno de los negocios}

Muchos estudiosos han señalado la influencia que el entorno de los negocios ejerce sobre el desempeño de las empresas. Burns y Stalker (1961) fueron los primeros en sistematizar una teoría acerca de los efectos del entorno sobre el desempeño corporativo. Examinaron cerca de 20 firmas industriales en el Reino Unido, concentrándose en cómo el patrón de las prácticas gerenciales en estas empresas se relacionaba con ciertas características de su entornoexterno. Los factores externos particulares que examinaron fueron las tasas de cambio de las técnicas científicas y los mercados de las industrias seleccionadas. Luego exploraron las relaciones entre las prácticas gerenciales internas y estas condiciones externas para descubrir su efecto sobre el descmpeño. Lawrence y Lorch (1985), haciendo un seguimiento de ese estudio clásico, entrevistaron a un grupo de gerentes norteamericanos sobre cómo lacreciente tasa de cambiotecnológico y la diversificación de las condiciones geográficas que surgían de la globalización de la firma afectaban su organización. Luego explicaron cómo estas circunstancias externas determinaban el tipo de organización que tendría éxito.

Austin (1990) examin6 específicamente el entorno de los negocios en los países en desarrollo y su efecto sobre las empresas extranjeras. Entre los factores que considera típicos del entorno de los negocios de estos países se encuentran: bajo nivel de tecnología, falta de información, enorme brecha entre los ingresos de los más pobres y los más ricos y gran centralización política. Los primeros dos factores dan como resultado una lenta tasa de cambio en las técnicas científicas locales; y el tercero, un mercado pequeño (debido a la injusta dis- tribución de los ingresos). El cuarto factor significa que el gobierno regula la mayor parte de la actividad económica: control de precios, de importaciones $y$, a veces, de divisas.

Frente a ese conjunto de factores ambientales hay posibilidades de solamente un pequeño número de relaciones de intercambio y un alto grado de incertidumbre. En esas circunstancias la empresa puede decidir pasar por alto el mercado y depender del uso de influencias. En un entorno protegido, las firmas tratan de aprovecharse del mismo y de incrementar en la medida de to posible el oportunismo (Williamson, 1975).

La evolución de las firmas venezolanas refleja la influencia de los factores ambientales descritos anteriormente. Las firmas familiares prevalecen porque han invertido una gran suma de dinero para sobreponerse a la infraestructura deficiente y a la falta de información (Austin, 1990). Han confiado en entablar contactos con personas influyentes del gobierno para ganar una mayor participación en el mercado tomando parte en el diseño de las políticas gubernamentales, como en las famosas políticas proteccionistas orientadas hacia la promoción de la industria local que impidieron la competencia extranjera a lo largo de tres décadas.

El entorno de negocios venezolano se caracteriza por la falta de criterios sobre imparcialidad y justicia. Venezuela es un país pequeño donde cierto medio es muy reducido: "todos se conocen". Quienes ocupan cargos gerenciales frecuentemente están vinculados por relaciones de parentezco con personas que tienen influencia en el gobierno. Por lo tanto, es común la existencia de una multiplicidad de pape- 
les, lo que a veces implica exigencias encontradas y lealtades en conflicto. Como resultado, a menudo hay conflicto de intereses, lo que dificulta ser imparcial y tener criterios claros sobre justicia y pureza administrativa (Naím, 1989).

Las grandes desigualdades económicas pueden generar descontento social e inestabilidad política. Ésta es una amenaza constantc, lo que afecta la planificación a mediano y largo plazo y obliga a los empresarios a entablar relaciones con personas influyentes dentro del gobierno, quienes pueden ayudarles a sacar el mayor beneficio en muy corto plazo.

Por otro lado, la debilidad del sistema financiero determina que los grupos empresariales locales a menudo deban crear sus propias instituciones financieras para compensar esa carencia. Los obstáculos para la obtención de crédito o recursos financieros obligan también a establecer contactos con personas del mundo financiero (Austin, 1990).

En los primeros años de la década de los ochenta, la fuga de capitales en los países en desarrollo, particularmente en Venezuela. supcraba por un tercio todo el capital que entraba, causando un serio deterioro en la posición de capital del país. El resultado fue controles de cambio y rigidez en la concesión de créditos (Austin, 1990). Las políticas gubernamentales obligaron al empresario a recurrir a conexiones personales con ciertos funcionarios del gobierno que les podían dar un trato especial para la adquisición de divisas y la obtención de apoyo financiero.

Los vínculos familiares son, con frecuencia, el elemento principal en la creación y determinación de los "grupos em- presariales", tan importantes como tipo de organización y como fuerza competitiva en los países en desarrollo. Estas redes crean una ventaja competitiva promoviendo la lealtad entre sus miembros y un mejor acceso a la información.

En Venezuela, la gran mayoría de empresas son familiares y están dirigidas por miembros de la familia propietaria o por personas muy vinculadas a ellos. Se diversificaron, primero, para obtener provecho de la escasa competencia que tuvieron por casi treinta años y, segundo, para acceder a recursos financieros. Esta forma es más flexible para afrontar las rigideces del entorno local de los negocios.

Entonces, la falta de información con relación a los competidores y la inestabilidad de las políticas públicas dificultan la planificación y la administración del crédito, lo cual, unido al deficiente sistema legal, ha llevado a los empresarios a confiar en que las relaciones personales faciliten su accionar. Esta manera de pensar y operar de los empresarios venezolanos está en contradicción con la cultura de la empresa multinacional estudiada, para la cual los negocios no deben regirse por cuestiones o víneulos personales.

\section{Resistencias a la cultura de la empresa}

Al encontrar diferencias entre su propia visión ética del contexto local y los valores de la empresa, los gerentes venezolanos desarrollan un sentimiento ambivalente. Por unia parte, aceptan la cultura corporativa porque la empresa les ofrece un empleo estable. relativamente bien remunerado y muchas oportunidades para prosperar; por otra parte, no se identifican con la cultura corporativa porque observan loque sucede 
fuera de la firma y entienden que los negocios no pueden regirse por reglas fijadasen un contexto económico y político tan diferente, como los Estados Unidos.

La ambigüedad entre la aceptación y la poca identificación con los valores de la casa matriz por los miembros de las subsidiarias lleva a pensar en la cultura corporativa como algo fragmentado, que incorpora tensión y conflicto y, al mismo tiempo, constituye un hecho que ayuda a entender lo que se ha avanzado sobre el tema.

Los estudios recientes sobre cultura organizacional ponen énfasis en las relaciones de poder al interior de la organización y en las formas de resistencia de los individuos, quienes en lugar de ser sujetos pasivos de la sociedad, cultura y socialización, reaccionan ante ellas. Estos estudios ayudan a entender lo descrito hasta aqui, específicamente las dos reacciones ambivalentes, la aceptación de los valores cor-porativos y la insatisfacción por el poco poder de decisión, como cultura y contracultura corporativas.

Para Smircich y Calas (1987), la cultura de una empresa no es estática ni uniforme, se teoriza como una expresión creativa de los habitantes de un escenario en particular, un proceso de simbolización que es amorfo, transitorio y sensual. El análisis organizacional intenta dar cuenta de las distintas estrategias que tienen sentido en el centro de trabajo.

Según Martin y Sichl (1983), la cultura está custodiada por la gerencia, es una expresión de autoridad y privilegio, es la gama simbólica a través de la cual se controlan las fuerzas desintegrativas y disfuncionales. Para Frost y otros (1991), la cultura organizacional consiste en múltiples interpretaciones que no llegan a formar una imagen clara. Para Alvesson (1984), la cultura organizacional expresa las desigualdades entre intereses, exponiendo los productos inevitables de la desarmonía. Alvesson y Willmott (1992) sostienen que los gerentes a cargo de organizaciones tienen interés en mantener las relaciones jerárquicas vigentes y usan distintas formas de discurso, tales como la cultura corporativa, para comunicar la legitimidad de sus propios intereses.

Los estudios etnográficos de Collinson (1993) y Kunda (1992) contribuyen a aclarar si la cultura era aceptada o no en la planta. Estudiaron la resistencia a campañas realizadas para promover la cultura corporativa. Collinson (1993) sostiene que la resistencia permanece como una característica persistente, significativa y notable de las organizaciones contemporáncas, la cual requiere un estudio crítico más detallado. Este autor identifica dos estrategias contrarias, influenciadas por distintas orientaciones subjetivas hacia el poder, el conocimiento y la información. La resistencia a través de la distancia es la manera como los subordinados intentan escapar o evitar las exigencias de la autoridad, y se alejan, obien físicamente osimbólicamente, de la organización y la estructura de poder prevaleciente. En contraste, en la resistencia a través de la persistencia, los subordinados buscan exigir una mayor participación en la organización y hacer que la gerencia esté más sujeta a rendir cuentas mediante la obtención de información. prácticas de monitoreo y desafiando procesos de toma de decisiones.

Para Kunda (1992), las culturas deben ser estudiadas en su contexto, considerando simultáneamente las concepciones gerenciales de la cultura, su aplicación y 
las respuestas de los miembros. A travếs de un estudio de observación participante, Kunda (1992) notóque mientras los gerentes tratan de "ligar los corazones y las mentes de los empleados" al interés corporativo, la respuesta más común del empleado es la de ironía -aceptación y distanciamiento al mismo tiempo-.

De manera muy parecida al caso de Kunda (1992), los gerentes de la empresa que aquí se analiza mostraron señales de aceptación y rechazo hacia la cultura corporativa. La aceptación se manifestaba en la iđentificación que la mayoría de los gerentes y empleados sentian hacia los fracasos y éxitos de la empresa -transmitidos a través de toda la organización vía correo electrónico-, así como la gran importancia que otorgaban al estar trabajando en una empresa prestigiosa. El rechazo se manifestaba principalmente en la preocupación de los gerentes por el hecho de que la empresa ignoraba su propio entendimiento del entorno de negocios venezolano.

Los gerentes encontraban apoyo para su desacuerdocon la cultura corporativaen su conocimiento del entorno de negocios local. Cuando se sentían presionados por el logro de las metas de la empresa, aun cuando ésta no podía ofrecerles posibilidades de mejora, los gerentes comentaban que la empresa no entendía, tan bien como ellos, la manera de hacer negocios en Venezuela: es decir, se separaban de la compañía manteniendo sus propias opiniones sobre cómo debían hacerse los negocios.

De cierta manera, lo encontrado en el presente estudio también guarda semejanza con la tesis de Adler (1992) de que la cultura organizacional no disminuye la importancia de la cultura nacional. La cul- tura nacional justificó cerca del 50 por ciento de las diferencias en las actitudes y cultura de los empleados (Hofstede, 1980). Adler (1992) explica que la presión por ajustarse a la cultura organizacional de una compañía extranjera provoca la resistencia del empleado y causa que éste se aferre más firmemente a su identidad nacional. Las presiones son mayores cuando los gerentes tienen que seguir luchando por los objetivos corporativos aun cuando la organización no puede satisfacer sus metas personales. En el caso descrito aquí, éste es el momento cuando los gerentes empiczan a separarse de la cultura corporativa.

Cómo influye el entorno de los negocios en las resistencias a la cultura de la empresa

Ya que los critcrios tradicionales guían las actividades de los grupos y organizaciones que predominan en el contexto empresarial venezolano, las personas son evaluadas según a quiénes conocen, no según sus capacidades y educación (Naím, 1989). Esto significa que aunque los gerentes desarrollen vínculos sociales y profesionales que pueden servirles para lograr una mejor posición, la firma no les ofrece posibilidad alguna de utilizarlos. Mientras los gerentes se adaptan a la cultura corporativa $y$ tratan de evitar confictos entre intereses contradictorios, ven su oportunidad para avanzar $y$ eligen los valores de la firma. Pero saben que éstos sólo son válidos dentro de la corporación, saben que el conocimiento técnico muy específico que han adquirido no les será útil fuera de ésta. Por esta razón, valoran la adquisición de conocimientos técnicos sólo hasta cierto punto.

Laadquisición de conocimiento nosignifica necesariamente que uno progresará 
en el contexto empresarial venezolano. De hecho, al comparar la muestra en estudio con su grupo de referencia (gerentes de la misma edad, tipo de educación y número de cargas familiares), se encontró que se pierde cierta cantidad de tiempo al adquirir conocimientos, ignorando, por una parte. la realidad del contexto de los negocios local, cspecíficamente los criterios tradicionales mediante los cuales se cvalúa las acciones de grupos $\mathrm{e}$ individuos, $\mathrm{y}$, por otra parte, la importancia de desarrollar las redes sociales que conducen al desarrollo profesional.

Entre la influencia proveniente del entorno de los negocios y la proveniente de la cultura corporativa se produce un conflicto que es casi imposible de resolver, porque ambas son válidas: la primera impulsa la batalla por sobrevivir en circunstancias poco controlables por la empresa; la segunda apunta hacia la construcción de su alta reputación; dos metas muy distintas, legítimas tanto para la organización como para sus miembros.

Se sabe que cuando existe desacuerdo entre los objetivos personales y los de la empresa, los empleados tienden a sentirse insatisfechos por las condiciones de trabajo y en ese momento empiezan a buscar oportunidades para mejorarlas. Obviamente, esto no es posible para todos los ejecutivos de alto nivel que en algún momento llegan a una posición desde la cual no pueden avanzar más. Algunos de ellos tendrán que quedarse, otros se retirarán cuando se sientan insatisfechos. Estos últimos habrán hecho contactos comerciales importantes y obtenido mucha experiencia práctica que les podrá servir para abrir su propio negocio o hacerse socio de alguno.

En el caso concreto de esta compañía multinacional -de servicios financieros-, los gerentes tienen pocas posibilidades de ofrecer grandes créditos a la industria porque la inversión extranjera, especialmente en el sector financiero, ha sido restringida por el gobierno venezolano. Por lo tanto, tienen pocas oportunidades de obtener ganancias para la firma, lo que a su vez significa pocas posibilidades de desarrollarse profesionalmente cerrando tratos importantes. Por una parte, el código de ética es tan estricto que excluye a muchos clientes con prácticas inapropiadas, utilizando criterios muy rígidos, clasificándolos en un "lista negra"; por otra parte, la actitud inadecuada del gobierno hacia la inversión extranjera limita las oportunidades que tienen los gerentes para identificarse con la cultura corporativadurante un periodo prolongado de tiempo.

Los gerentes pueden ignorar el entorno de los negocios siempre y cuando la firma les esté ofreciendo la oportunidad de avanzar profesionalmente. Mientras eso sucede, se adaptan a la cultura corporativa principalmente para evitar un posible conflicto entre los papeles que cumplen dentro de la empresa y sus propias metas, como darle mayor prioridad a obtener ingresos antes que respetar el código ético de la empresa. Empiezan a prestarle más atención al entorno local de los negocios cuando la firma no puede satisfacer sus metas profesionales y personales. En ese momento toman conciencia de que fuera de la cmpresa no se valora recibir un entrenamiento tan especializado. La corporación utiliza procesos tecnológicos sofisticados para sus operaciones comerciales, lo que le da una ventaja competitiva con relación a las empresas locales del mismo sector. Pero la preparación técnica recibida por los gerentes en la firma es muy específica, es aplicable únicamente dentro de ella. 
Por esa razón, la inversión extranjera puede ser un problema en vez de una solución en cuanto al desarrollo tecnológico. Las destrezas técnicas requeridas dentro de una firma multinacional son útiles solamente para ese tipo de firmas. No son una ventaja para competir en el mercado laboral local, donde las filiaciones son más importantes que el entrenamiento que uno haya recibido.

A pesar de lo mencionado en el párrafo anterior, la inversión extranjera podría generar nuevas oportunidades para los gerentes venezolanos que hayan desarrollado habilidades técnicas. Si se expandiera la inversión extranjera, individuos altamente capacitados podrían optar por posiciones en empresas multinacionales y mixtas, que valoran tanto el entrenamiento como la supervisión continua desde las casas matrices ubicadas en los países desarrollados.

Ciertos factores ambientales contribuyen a que la inversión extranjera se expanda muy lentamente. Por ejemplo, un entorno altamente politizado, es decir, un alto gradode centralización política. De la misma manera, el precario sistema judicial, que no ofrece garantía alguna de que un cliente irresponsable cumpla con sus obligaciones financieras. Estos mismos factores del entorno de los negocios contribuyen al hecho de que la cultura de la firma sea, a veces, inflexible, pues la ayuda a protegerse de los riesgos que entrañan estas dificultades.

La cultura corporativa, como conjunto de valores y creencias con relación a lo que es importante y "la forma como se hacen las cosas en la firma" genera un mecanismo de integración entre los empleados que fortalece a quienes se identifican con ella. El entorno local de los negocios ejerce una fuerte influencia, aunque latente, que moldea las expectativas de los gerentes. La empresa multinacional ha diseñado un instrumento que aísla a su personal $-\mathrm{y}$ a sí misma-del entorno de los negocios local, por lo menos durante años importantes de la carrera profesional de un gerente, $y$ en esto consiste precisamente la cultura corporativa. Las decisiones más importantes se toman lejos del entorno nacional de negocios, $\mathrm{y}$ aun así las personas se sienten comprometidas con la organización y forman sus expectativas según lo que ésta les ofrece. 


\section{Referencias Bibliográficas}

ADLER, Nancy. Intemational dimensions of organizational behaviour. Boston: Wadsworth Inc, 1992.

ALVESSON, Matts. On the idea of organizational structure. Ponencia presentada a la First International Conference on Organizational Symbolism and Corporate Culture.UnivensidaddeLund.Suecia, 1984.

ALVESSON, Matts y WILMOTT, Hugh. Critical management studies. Londres: Sage Publications, 1992.

AUSTIN, James. Managing in developing countries: strategic analysis and operating techniques. Nueva York: The Free Press, 1990.

BURNS. Tom y STALKER, G.M. The management of innovation. Londres: Tavistock Publications, 1961.

COLLINSON, David. Knowledge, resistence and power. Ponencia presentada al Decimoprimer coloquio del Grupo Europeo de Estudios de Organización (EGOS). París, julio, 1993.

DUTTON, Janey DUKERICH,Jane. "Keeping an cyc on the mimor image of identity in organizational adaptation". Academy of Management Journal, 34 (3): 517-554, 1992.

ELSBACH, Kinmberly y SUTTON, Robert. "Acquiring organizational legitimacy through illegitimate actions: a marriage of institucional and impression management". Academy of Management Journal, 35 (4): 699-738, 1992.

FRANCÉS, Antonio. "El entomo de las corporacionesen Venezue!a".69-97 pp. En Francés, A. La corporación en 4 dimtensiones. Caracas: Ediciones IESA, 1992.
FROST. Peter; MOORE, Larry: LOUIS, Meryl R.:LUNDBERG, Craigy MARTIN, Joanne (Eds.). Reframing organizational culture. Londres: Sage Publications. 1991.

HOFSTEDE, Geert. Culture's consequences: international differences in work related values. Beverly Hills: Sage Publications. 1980.

KANTER. Rosabeth Moss. Men and women of the corporation. Nueva York: Basie Books. 1977.

KUNDA, Gideon. Engineering culture: con. trol and commitments in a high tech corporation. Filadelfia: Temple University Press, 1992.

LAWRENCE, Paul y LORCH, Jay W. Organizational enviroment: managing differentiation and integration. Boston: Harvard University Press, 1985.

MARTIN, Joanne y SIEHL, Caren. "Organizational culture and counter culturc". Organizational Dynamics, 12 (2): 52.64. 1983.

NAIM, Moisés. "Viejas costumbres y nuevas realidades en la gerencia venezolana". 493 . 520 pp. En Naím, M (Ed.). Les empresas venezolanas: su gerencin. Caracas: Ediciones IESA. 1989.

PÉREZ.PERDOMO, Rogelio. "Corrupción y entorno de negocios en Venezuela", 3-26 pp. En Perdomo R. y Capriles. R. (Eds.). Corrupción y control en Venezucla: una perspectiva comparada. Carncas: Ediciones IESA, 1991.

SMIRCICH, Linda y CAL.AS. Martha. "Organizational culture: a critical assesment". 228-263 pp. Lin Jablia, F.; Oantam, L.: Roberts, K. y Porter. L. (Eds.). 
Handbook of organizational communication: an interdisciplinary approch. Newbury Park: Sage Publications, 1987.

WATSON, Tony. In search of management. Londres: Routledge Books, 1994.
WILLIAMSON, Oliver. Markets and hierarchies: analysis and anti-trust implications. Nueva York: The Free Press, 1975. 